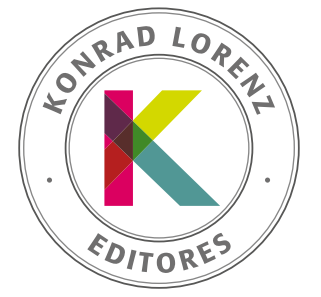

\title{
Efecto del uso de estrategias cognitivas de regulación emocional sobre la ansiedad en adolescentes españoles
}

\author{
Macarena del Valle ${ }^{a, *}$, Elena Betegón ${ }^{\mathrm{b}}$ y María Jesús Irurtia
}

\begin{abstract}
a Universidad Nacional de Mar del Plata -UNDMP- - Instituto de Investigación en Psicología Básica, Aplicada y Tecnología -IPSIBAT- Consejo Nacional de Investigaciones Científicas y Técnicas -CONICET-, Mar del Plata, Argentina

${ }^{b}$ Dpto. Psicología. Personalidad, Evaluación y Tratamiento Psicológico - Universidad de Valladolid -UVA-, Valladolid, España
\end{abstract}

Recibido el 31 de octubre de 2018; aceptado el 11 de enero de 2019

\author{
PALABRAS CLAVE \\ regulación \\ emocional, \\ ansiedad, \\ género, estudiantes \\ de nivel secundario, \\ psicopatología
}

\begin{abstract}
Resumen La forma en la que las personas regulan sus emociones suele influir en la aparición, curso y desarrollo de un amplio espectro de psicopatologías. El objetivo de este estudio fue evaluar el efecto del uso de las estrategias cognitivas de regulación emocional sobre el nivel de ansiedad de estudiantes de nivel secundario. Para ello se aplicó la adaptación al castellano del CER-Q y la adaptación española del STAI a una muestra de 266 estudiantes de nivel secundario. Se planteó un modelo de regresión lineal múltiple, donde el nivel de ansiedad fue la variable dependiente, y la frecuencia de uso de estrategias de regulación emocional fueron las variables independientes. El género también fue considerado una variable independiente puesto que los datos mostraron diferencias en el nivel de ansiedad y en el uso de estrategias de regulación emocional entre sujetos femeninos y masculinos. El modelo explicó un $46.3 \%$ de la varianza del nivel de ansiedad, y las variables que resultaron predictoras fueron autoculparse, reinterpretación positiva, catastrofización, rumiación y género. En función de los hallazgos y de la literatura previa, se apunta a la idea de que el uso de estrategias adaptativas de regulación emocional promueve menores niveles de ansiedad y el uso de estrategias desadaptativas redunda en mayores niveles de ansiedad.

(c) 2018 Fundación Universitaria Konrad Lorenz. Este es un artículo Open Access bajo la licencia CC BY-NC-ND (http://creativecommons.org/licenses/bync-nd/4.0/).
\end{abstract}

Effect of the use of cognitive emotional regulation strategies on anxiety levels in Spanish adolescents

\begin{abstract}
The way in which people regulate their emotions is related to the appearance, course and development of a broad spectrum of psychopathologies. The aim of this study was to assess the effect of the use of cognitive emotional regulation strategies on high school students' anxiety level. To this end, the Spanish adaptation of the CER-Q and the Spanish adaptation of the STAI were applied to a sample of 266 high school students. A multiple linear regression model was proposed, where anxiety level was the dependent variable, and the use
\end{abstract}

\author{
emotional regulation, \\ anxiety, gender, \\ high school students, \\ psychopathology
}

* Autor para correspondencia.

Correo electrónico: mdelvalle1989@gmail.com. 
frequencies of emotional regulation strategies were the independent variables. Gender was also considered as an independent variable since the data showed differences in anxiety level and in the use of emotional regulation strategies between male and female subjects. The model explained $46.3 \%$ of the variance, and the predictive variables were Self-blame, Positive Reinterpretation, Catastrophizing, Rumination and gender. Based on the findings and the previous literature, it is suggested that the use of adaptive strategies of emotional regulation promotes lower levels of anxiety, and the use of maladaptive strategies results in higher levels of anxiety.

(c) 2018 Fundación Universitaria Konrad Lorenz. This is an open access article under the CC BY-NCND license (http://creativecommons.org/licenses/bync-nd/4.0/).

La adolescencia es un periodo de grandes cambios que se suceden en un breve lapso de tiempo (Díaz Santos \& Santos Vallín, 2018). En España, en particular, los adolescentes se encuentran sometidos a diversas presiones académicas y decisiones vitales a edades tempranas. Alrededor de los 15 años, finalizando la educación secundaria obligatoria (ESO), deben optar por continuar con el bachillerato $-\mathrm{y}$ avanzar hacia la universidad-, o elegir una formación profesional. Y para aquellos que se decidan por el bachillerato, se inicia una etapa adicional de exigencias ligadas a los procesos de selectividad universitaria.

En la necesidad de adaptarse a los nuevos cambios en el desarrollo inherentes a esta etapa vital y a las demandas académicas mencionadas, suelen surgir en la adolescencia distintas alternaciones entre las cuales se encuentran, más comúnmente los trastornos de ansiedad (Orgilés, Méndez, Espada, Carballo, \& Piqueras, 2012). Este tipo de psicopatología afecta de manera directa el bienestar de los adolescentes y su funcionamiento social, emocional y académico (e.g., Hernández-Pozo, Ramírez-Guerrero, López-Cárdenas, \& Macías-Martínez, 2015); además, predice la aparición y desarrollo de trastornos similares en la adultez (Copeland, Angold, Shanahan, \& Costello, 2014).

La ansiedad es una emoción y, por lo tanto, se manifiesta en la experiencia subjetiva, en la activación psicofisiológica y en la conducta (Mulligan \& Scherer, 2012). En sí misma, es una respuesta adaptativa; pero si se presenta en el momento equivocado, o con un nivel de intensidad inadecuado, puede resultar disruptiva, perjudicar los procesos cognitivos, interferir con la adaptación al ambiente y afectar el rendimiento académico (Domínguez-Lara, Alarcón-Parco, \& Navarro-Loli, 2017; Gross, 2014; Jacobs \& Gross, 2014; Oros, Manucci, \& Richaud-de Minzi, 2011). En consecuencia, es necesario en ocasiones un proceso complementario de regulación de la emoción (Pekrun \& Linnenbrick-García, 2012). La regulación emocional supone la activación de diversos mecanismos orientados a evaluar y modificar un estado afectivo en curso, es decir, al ejercicio consciente de la regulación de la emoción (Koole, 2009). Garnefksi, Kraaij y Spinhoven (2001) proponen la existencia de nueve estrategias que nos permitirían regular nuestras emociones a partir de nuestros procesos cognitivos. Estas se detallan en la tabla 1. Según el modelo, y de manera general, las primeras cuatro estrategias resultarían desadaptativas en términos de su utilidad para alcanzar objetivos específicos, y las últimas cinco serían adaptativas.

Ahora bien, la regulación emocional ha sido incorporada como una variable explicativa en los modelos de psicopatología (Cisler, Olatunji, Feldner, \& Forsyth, 2010; Sheppes, Suri, \& Gross, 2015). La literatura propone que cuando las personas no son capaces de regular sus emociones de una manera efectiva, el malestar persiste en el tiempo, lo que puede provocar la aparición de sintomatología de diverso tipo (Aldao, Nolen-Hoeksema, \& Schweizer, 2010; Cano-Vindel, 2011; Hervás, 2011). En particular, el uso frecuente de estrategias de regulación emocional desadaptativas (e. g., rumiación, Nolen-Hoeksema, Wisco, \& Lyubomirsky, 2008), o el uso poco frecuente de estrategias adaptativas (e.g., reevaluación, Eftekhari, Zoellner, \& Vigil, 2009) ha sido asociado a mayores niveles de ansiedad.

No obstante, los estudios reportan en ocasiones resultados poco consistentes respecto de qué estrategias de regulación emocional resultan predictoras de los niveles de ansiedad. Garnefski, Legerstee, Kraaij, Van den Kommer y Teerds (2002), evaluaron a estudiantes de nivel secundario y hallaron que, entre los adolescentes, de las nueve estrategias de regulación emocional del modelo de Garnefksi et al. (2001), solo rumiación y autoculparse aportaban a la varianza explicada de los niveles de ansiedad. Luego, Yousefi (2007) realizó un estudio de características similares con escolares iraníes de entre 7 y 9 años y halló que las estrategias de regulación emocional que predecían los niveles de ansiedad de los alumnos eran autoculparse, rumiación, reinterpretación positiva, catastrofización y culpar a otros. Legerstee, Garnefski, Verhulst y Utens (2011) hallaron que en adolescentes de 12 a 16 años, la estrategia de reinterpretación positiva no resultó predictora de los diferencias en los niveles de ansiedad, pero sí lo eran rumiación, catastrofización y autoculparse. En un estudio más reciente, Potthoff et al. (2016) realizaron una investigación transcultural, en la que participaron estudiantes universitarios y personas de población general provenientes de Alemania, Países Bajos, Hungría, España, Italia y Portugal. Los autores hallaron que solo la estrategia de catastrofización predecía de manera directa la ansiedad en las muestras de todos los países. Todas las estrategias de regulación emocional restantes presentaron algún tipo de asociación, pero estas variaban en presencia e intensidad en función del país de procedencia de los sujetos.

En el contexto iberoamericano, Domínguez-Lara (2017) encontró en estudiantes universitarios que las estrategias que más predecían los niveles de ansiedad eran nuevamente rumiación, catastrofización y culpar a otros, aunque también autoculparse aportó a la varianza explicada. Por su parte, Andrés, Castañeiras y Richaud de Minzi (2015) reportan que las estrategias autoculparse, rumiación y reinterpretación positiva son las que resultaron predictoras de los niveles de ansiedad de niños de 10 años, no así catastrofización ni culpar a otros. Como puede observarse, aún hay discrepancias respecto a qué estrategias de regulación emocional resultan predictoras de los niveles de sintomatología ansiosa. 
Tabla 1 Descripción resumida de las estrategias cognitivas de regulación emocional según el modelo de Garnefski, Kraaij y Spinhoven (2001) y Garnefski y Kraaij (2007)

\begin{tabular}{|c|c|}
\hline Estrategia & Descripción resumida \\
\hline Culpar a otros & Refiere a los pensamientos en donde la culpa por la experiencia vivida reside en los demás. \\
\hline Autoculparse & $\begin{array}{l}\text { Refiere a los pensamientos en donde la persona se culpa a sí misma por el evento negativo expe- } \\
\text { rimentado. Esta estrategia desadaptativa no debe interpretarse como la toma de responsabilidad } \\
\text { sobre una situación, sino como la puesta del foco atencional en las emociones y pensamientos } \\
\text { negativos ligados a la culpa, como la ansiedad y el enojo. }\end{array}$ \\
\hline Rumiación & $\begin{array}{l}\text { Supone focalizarse sistemática y excesivamente en los pensamientos y sentimientos negativos } \\
\text { asociados al evento displacentero en cuestión. }\end{array}$ \\
\hline Catastrofización & $\begin{array}{l}\text { Consiste en exacerbar la negatividad del evento experimentado. Las personas que utilizan esta es- } \\
\text { trategia suelen enfatizar y magnificar las emociones y pensamientos negativos ligados a un suceso } \\
\text { y tienden a considerar que lo que aconteció es lo peor que podría haber ocurrido. }\end{array}$ \\
\hline Poner en Perspectiva & $\begin{array}{l}\text { Implica tener pensamientos que disminuyan o reduzcan la gravedad o seriedad del evento negati- } \\
\text { vo, relativizándolo y comparándolo con otros. }\end{array}$ \\
\hline Reinterpretación positiva & $\begin{array}{l}\text { Supone tener pensamientos que dan un nuevo significado positivo al suceso displacentero, inter- } \\
\text { pretándolo como una nueva oportunidad de crecimiento personal. }\end{array}$ \\
\hline Focalización en los planes & $\begin{array}{l}\text { Refiere a los pensamientos sobre la forma y los pasos a seguir para manejar el evento displacen- } \\
\text { tero. No implica necesariamente que la planificación se traduzca en actos, sino que refiere a la } \\
\text { producción de procesos cognitivos ligados a las formas para hacer frente a una situación. }\end{array}$ \\
\hline Focalización positiva & $\begin{array}{l}\text { Consiste en generar pensamientos sobre tópicos alegres y placenteros en lugar de pensar sobre el } \\
\text { evento problemático. }\end{array}$ \\
\hline Aceptación & $\begin{array}{l}\text { Refiere a los pensamientos ligados a aceptar el suceso negativo y resignarse a lo que ha aconteci- } \\
\text { do. Aceptar la realidad de la situación pasada supone, en cierta forma, un avance hacia el intento } \\
\text { de lidiar con ella. }\end{array}$ \\
\hline
\end{tabular}

Además, el género también reviste interés en este modelo explicativo, puesto que distintos estudios sugieren que las personas de género femenino tienden a exhibir mayor prevalencia de sintomatología ansiosa y desórdenes de ansiedad (Caballo et al., 2014, 2016; Irurtia, Caballo, \& Ovejero, 2009; McLean, Asnaani, Litz, \& Hofmann, 2011; Orgilés et al., 2012), diferencia que suele surgir incluso desde la niñez (Bender, Reinholdt-Dunne, Esbjørn, \& Pons, 2012), sostenerse en la adolescencia (Ranta et al., 2007) y mantenerse luego a lo largo de la vida (Leach, Christensen, Mackinnon, Windsor, \& Butterworth, 2008; Xu et al., 2012). Algunos estudios (e. g., Garnefski, Teerds, Kraaij, Legerstee, \& Van den Kommer, 2004) hallaron que las mujeres hacen un uso más frecuente de ciertas estrategias de regulación emocional como rumiación o catastrofización. En función de ello, Nolen-Hoeksema (2012) sugiere que un mayor uso de la estrategia rumiación por parte de las mujeres explicaría en parte los mayores niveles de ansiedad reportados por ellas.

No obstante, los estudios que han evaluado la existencia de diferencias en función del género en la frecuencia de uso de las distintas estrategias de regulación emocional del modelo de Garnefski et al. (2001), también han presentado resultados inconsistentes. Medrano, Moretti, Ortiz y Pereno (2013) hallaron un uso más frecuente de la estrategia poner en perspectiva por parte de las mujeres, pero ausencia de diferencias para el resto de las estrategias. Zlomke y Hahn (2010) también reportan un mayor uso de la estrategia poner en perspectiva en las participantes de su estudio, pero además hallaron diferencias en las estrategias rumiación y cul- par a otros, siendo la primera más frecuente en las mujeres y la segunda más frecuente entre los hombres. De la misma manera, Öngen (2010) encuentra mayores niveles de rumiación en las mujeres, pero sus resultados indican también un mayor uso por parte de los hombres de focalización positiva, focalización en los planes y reinterpretación positiva.

En función de lo expuesto, este estudio tuvo por objeto evaluar la existencia de diferencias en el uso de estrategias cognitivas de regulación emocional en función del género de los sujetos para aportar evidencias que permitan zanjar estas discrepancias. Además, se buscó evaluar el efecto del género y de la frecuencia de uso de las distintas estrategias cognitivas de regulación emocional, sobre los niveles de ansiedad para determinar cuáles de estas variables resultaban predictoras o explicativas de esta última.

\section{Método}

\section{Participantes}

La muestra fue de conveniencia, no probabilística y estuvo compuesta por un total de 266 participantes de entre 15 y 18 años $(M=15.71 ; D T=.77)$ de cuarto de educación secundaria obligatoria (ESO) $(n=152 ; 57.1 \%)$ y primero de bachillerato $(n=114 ; 42.9 \%$ ) de dos colegios concertados de la ciudad de Valladolid, España. Ambos colegios son concertados, no públicos tienen acceso gratuito a la ESO; mientras que el ciclo de bachillerato abona cuotas accesibles. El $50.4 \%$ de la muestra fue de género femenino $(n=134)$ 
y el $49.6 \%$ fue de género masculino $(n=132)$. Los colegios se encuentran establecidos en zonas urbanas y el nivel socioeconómico de los participantes, aunque no fue evaluado individualmente, fue reportado como medio a medio-alto de manera general por los directivos de la institución.

\section{Medidas}

Para evaluar la frecuencia de uso de estrategias cognitivas de regulación emocional, se utilizó la adaptación al castellano realizada por Medrano et al. (2003) del Cuestionario de Regulación Emocional y Cognitiva (CER-Q) desarrollado por Garnefski y Kraaij (2007) para evaluar los componentes cognitivos de la regulación emocional. El instrumento consta de una escala autoadministrada de 36 ítems que representan afirmaciones sobre pensamientos que suelen surgir en las personas frente a sucesos negativos, tales como "pienso cómo puedo afrontar mejor la situación" o "pienso que esa situación tiene también partes positivas". Los ítems exploran las nueve estrategias cognitivas de regulación emocional del modelo de Garnefski et al. (2001) y dan lugar a nueve escalas correspondientes, compuestas por cuatro ítems cada una: culpar a otros, autoculparse, aceptación, rumiación, catastrofización, poner en perspectiva, reinterpretación positiva, focalización en los planes y focalización positiva. La instrucción que se transmite a los sujetos es la siguiente: "Generalmente todos hemos tenido que afrontar ahora o en el pasado sucesos negativos o desagradables. Cada persona responde de una forma característica y propia ante estas situaciones. Por medio de las siguientes afirmaciones y en función de la siguiente escala de frecuencia, señale en qué piensa o qué siente cuando experimenta sucesos negativos o desagradables". La escala es de tipo Likert y las opciones de respuesta van de 1 "(casi) nunca" a 5 “(casi) siempre”. Los índices de consistencia interna (alfa de Cronbach) hallados en distintas investigaciones (e.g., Domínguez-Lara \& Medrano, 2016) y en las adaptaciones del instrumento a distintos países como Francia (Jermann, Van der Linden, d'Acremont, \& Zermatten, 2006), China (Zhu et al., 2008), España (Domínguez-Sánchez, Lasa-Aristu, Amor, \& Holgado-Tello, 2013) y Argentina (Medrano et al., 2013), oscilan entre los .60 y .90 puntos, replicando en los estudios la estructura de 9 factores original.

Si bien no existen estudios sobre las propiedades psicométricas de esta escala en población adolescente, la adaptación del instrumento fue validada en población joven desde los 18 años. Además, existen antecedentes de la utilización en población adolescente (de 12 a 18 años) de otras versiones del instrumento desarrolladas o adaptadas en población de mayor edad (e.g., Duarte, Matos, \& Marques, 2015; Garnefski, Kraaij, \& Van Etten, 2005). No obstante, como medida de confiabilidad, se presenta a continuación el coeficiente alfa de Cronbach para las subescalas: culpar a otros $=.76$; autoculparse $=.72$; rumiación $=$ .74 ; catastrofización $=.71$; reinterpretación positiva $=.71$; poner en perspectiva $=.73$; focalización en los planes $=.61$; refocalización positiva $=.81$; aceptación $=.57$. Los índices fueron entre aceptables y buenos, a excepción del hallado en la dimensión aceptación. En este caso, se identificó que la eliminación del ítem 25 ("Pienso que no puedo cambiar nada el respecto”) aumentaba la confiabilidad de la subescala a .72. Este aspecto se retoma en la discusión con la literatura.
Para medir el nivel de ansiedad se han utilizado los 20 reactivos correspondientes a la subescala de ansiedad-rasgo de la versión española (Spielberger, Gorsuch, Lushene, \& Cubero, 1999) del Cuestionario de ansiedad estado-rasgo (State Trait Anxiety Inventory [STAI]) (Spielberger, Gorsuch, \& Lushene, 1970). El STAI es una escala de autoinforme compuesta por 40 ítems en total, que evalúan de forma diferenciada la ansiedad como un estado o condición transitoria y la ansiedad como un rasgo o propensión estable. Dados los objetivos del estudio, se optó por trabajar únicamente con los ítems correspondientes a la ansiedad-rasgo, cuya consigna de evaluación general de la ansiedad resulta más acorde con aquella utilizada en el CERQ. Además, la ansiedad-estado resulta mucho más lábil y está ligada a elementos contextuales específicos, lo cual excede los objetivos de esta investigación.

En los 20 ítems correspondientes a la evaluación de la ansiedad-rasgo, el participante debe responder en una escala de 0 al 3 (de "casi nunca" a "casi siempre"), en qué medida se siente de esa manera generalmente. Ejemplos de ítems son "me preocupo demasiado por cosas sin importancia" o "me siento bien". El instrumento es ampliamente utilizado, resulta breve, sencillo y útil para la evaluación de la ansiedad. Respecto a su fiabilidad en población española, los valores alfa de Cronbach oscilan entre .84 y .93 (Fonseca-Pedrero, Paino, Sierra-Baigrie, Lemos-Giráldez, \& Muñiz, 2012; Guillén-Riquelme, \& Buela-Casal, 2011). El valor de alfa en nuestra muestra fue de .87 .

\section{Procedimiento y consideraciones éticas}

Se trata de un estudio explicativo, con un diseño no experimental, transversal (Hernández Sampieri, Fernández Collado, \& Baptista Lucio, 2014). Se siguieron cuidadosamente los lineamientos éticos internacionales designados por la American Psychological Association y la Declaración de Helsinki, así como los principios éticos respecto de las investigaciones en seres humanos, asegurando la confidencialidad de los datos y el actuar siempre para el beneficio de los participantes.

Para la toma de datos, los padres o tutores dieron su consentimiento informado para la evaluación y los adolescentes participaron del estudio voluntariamente. Los protocolos fueron administrados en sesiones grupales de entre 20 y 30 alumnos, donde los mismos estaban divididos en sus cursos habituales dentro de sus aulas. En todo momento hubo un evaluador presente para despejar las dudas que pudieran surgir. Se recolectaron asimismo los datos respecto al género.

\section{Análisis de los datos}

Las respuestas a los instrumentos fueron sistematizadas en una base de datos y sometidas a análisis estadísticos. Para caracterizar la muestra bajo estudio se utilizaron análisis estadístico-descriptivos. Se analizó la existencia de diferencias estadísticamente significativas en función del género mediante la aplicación de pruebas $t$ de Student y $U$ de Mann-Whitney, según correspondía, de acuerdo con el tipo de distribución y los criterios de normalidad. A continuación, para evaluar el tamaño del efecto de las diferencias que han resultado estadísticamente 
significativas, se han normalizado los índices de las variables con distribución anormal y se ha calculado la $d$ de Cohen (Ledesma, Macbeth, \& Cortada de Kohan, 2008).

Para evaluar las relaciones entre el uso de estrategias de regulación emocional y el nivel de ansiedad, se realizaron correlaciones parciales controlando el efecto del género (puesto que se hallaron diferencias en función de esta variable). Finalmente, se evaluó un modelo de análisis de regresión lineal múltiple (método enter), en donde la variable dependiente fue el nivel de ansiedad-rasgo, y las variables independientes introducidas fueron las estrategias cognitivas de regulación emocional y el género. Para considerar la aplicabilidad de la prueba de regresión se evaluó la normalidad de los residuos del modelo propuesto, hallando una distribución adecuada (Shapiro-Wilk = .992; $p=.13$ ). También fue realizado un diagnóstico de colinealidad (VIF), hallando valores inferiores de 1.94 en todos los casos.

\section{Resultados}

En principio, se determinó si existían diferencias estadísticamente significativas entre las participantes de género femenino y los de género masculino para las variables en estudio. Las estrategias de aceptación, catastrofización, poner en perspectiva, reinterpretación positiva y focalización en los planes han cumplido tanto con el criterio de normalidad como con el de homocedasticidad, por lo que se ha optado por la aplicación de una prueba $t$ de diferencia de medias. Los resultados de la prueba han indicado ausencia de diferencias estadísticamente significativas entre los grupos. Respecto del resto de las variables, es decir, culpar a otros, autoculparse, rumiación, focalización positiva y ansiedad-rasgo, estas no han cumplido, o bien el criterio de normalidad, o bien el de homocedasticidad, por lo que se ha optado por una prueba $U$ de Mann-Whitney. Los resultados de la prueba y el tamaño del efecto de las diferencias ( $d$ de Cohen) se muestran en la tabla 2.

Como puede observarse, las participantes de género femenino realizan un uso más frecuente de las estrategias autoculparse y rumiación, con diferencias pequeñas, mientras que los hombres hacen un uso más frecuente de la estrategia culpar a otros, siendo esta diferencia mediana. Además, las mujeres muestran un mayor nivel de ansiedad-rasgo, siendo la diferencia también pequeña.
Para evaluar las relaciones entre el nivel de ansiedad y la frecuencia de uso de estrategias cognitivas de regulación emocional, se han realizado, en primer lugar, correlaciones parciales, controlando el efecto del género. Los resultados se expresan en la tabla 3.

Se observó que el uso de estrategias desadaptativas (culpar a otros, autoculparse, rumiación, catastrofización) se asoció significativamente y de manera directa con mayores niveles de ansiedad ( $p<.05)$, indicando que mientras mayor es la tendencia de la persona a utilizar estrategias desadaptativas para regular sus emociones frente a un evento negativo o estresante, mayores son los niveles de ansiedad. Además, el uso frecuente de ciertas estrategias adaptativas, en particular, reinterpretación positiva y focalización positiva, se asoció de manera inversa con los niveles de ansiedad $(p<.01)$, lo que sugiere que el uso frecuente de estas estrategias de regulación emocional consideradas adaptativas se relaciona con menores valores autorreportados de ansiedad.

En segundo lugar, se ha efectuado una regresión lineal múltiple (método enter) respecto del efecto del género y el uso de estrategias de regulación emocional sobre la ansiedad. Los resultados se muestran en la tabla 4.

Se observa que el modelo resultó significativo $(p<.01)$, explicando un $46 \%$ de la varianza. Los beta estandarizados indican que las estrategias autoculparse, catastrofización y reinterpretación positiva aportan la mayor varianza explicada. Las variables rumiación y género, por su parte, resultaron también predictoras, pero su aporte fue menor.

\section{Discusión}

En principio, los resultados hallados en esta investigación respecto de las diferencias de género en los niveles de ansiedad son similares a lo ya expuesto en la literatura, coincidiendo con lo enunciado por otros autores como Blanco et al. (2006), Ranta et al. (2007), McLean et al. (2011), Bender et al. (2012), Xu et al. (2012), y Orgilés et al. (2012). Muchas investigaciones atribuyen estas diferencias a patrones culturales y de crianza divergentes para hombres y mujeres (e.g. Caballo et al., 2016). McLean y Anderson (2009), no obstante, interpretan estas diferencias como el resultado de la interacción de distintos factores, no solo

Tabla 2 Prueba U de Mann-Whitney para las variables que no cumplen el criterio de normalidad, sobre las diferencias en función del género en el uso de estrategias cognitivas de regulación emocional y el nivel de ansiedad

\begin{tabular}{|c|c|c|c|c|c|c|}
\hline & \multicolumn{2}{|c|}{ Masculino } & \multicolumn{2}{|c|}{ Femenino } & \multirow{2}{*}{$u$} & \multirow{2}{*}{$d$} \\
\hline & M & DT & M & DT & & \\
\hline Culpar a otros & 10.43 & 3.05 & 8.73 & 2.95 & $5979.50^{* *}$ & .57 \\
\hline Autoculparse & 12.73 & 2.76 & 13.40 & 2.88 & $7342.00^{*}$ & -.24 \\
\hline Rumiación & 12.82 & 3.27 & 14.23 & 3.51 & $6736.50^{* *}$ & -.42 \\
\hline Focalización positiva & 12.26 & 3.41 & 11.68 & 4.03 & 8007.00 & 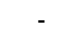 \\
\hline Ansiedad-rasgo & 20.85 & 8.60 & 24.71 & 9.30 & $6801.00^{* *}$ & -.43 \\
\hline
\end{tabular}

${ }^{*} p<.05$

${ }^{* *} p<.01$ 
Tabla 3 Correlaciones parciales entre la frecuencia de uso de estrategias cognitivas de regulación emocional y el nivel de ansiedad, controlando el efecto del género

\begin{tabular}{|c|c|c|c|c|c|c|c|c|c|c|}
\hline & 1 & 2 & 3 & 4 & 5 & 6 & 7 & 8 & 9 & 10 \\
\hline 1. Culpar a otros & & .08 & .04 & $.18^{* *}$ & $.41^{* *}$ & $.27^{* *}$ & $.10^{*}$ & $.11^{*}$ & $.21^{* *}$ & $.12^{*}$ \\
\hline 2. Autoculparse & & & .09 & $.41^{* *}$ & $.41^{* *}$ & .03 & -.06 & $.13^{*}$ & $-.15^{* *}$ & $.48^{* *}$ \\
\hline 3. Aceptación & & & & $.15^{* *}$ & -.09 & $.34^{* *}$ & $.28^{* *}$ & $.31^{* *}$ & $.26^{* *}$ & .01 \\
\hline 4. Rumiación & & & & & $.42^{* *}$ & $.14^{* *}$ & .03 & $.27^{* *}$ & .04 & $.41^{* *}$ \\
\hline 5. Catastrofización & & & & & & .01 & $-.19^{* *}$ & -.08 & -.07 & $.50^{* *}$ \\
\hline 6. Poner en perspectiva & & & & & & & $.51^{* *}$ & $.36^{* *}$ & $.37^{* *}$ & -.08 \\
\hline 7. Reinterpretación positiva & & & & & & & & $.55^{* *}$ & $.44^{* *}$ & $-.33^{* *}$ \\
\hline 8. Focalización en los planes & & & & & & & & & $.29^{* *}$ & -.08 \\
\hline 9. Focalización positiva & & & & & & & & & & $-.17^{* *}$ \\
\hline 10. STAI & & & & & & & & & & \\
\hline
\end{tabular}

${ }^{*} p<.05$

${ }^{* *} p<.01$

Tabla 4 Modelo de análisis de regresión lineal múltiple por pasos con el nivel de ansiedad como variable dependiente

\begin{tabular}{|c|c|}
\hline Variable predictora & $\beta$ \\
\hline Género & $-.14^{* *}$ \\
\hline Culpar a otros & -.03 \\
\hline Autoculparse & $.27^{* *}$ \\
\hline Aceptación & .06 \\
\hline Rumiación & $.19^{* *}$ \\
\hline Catastrofización & $.26^{* *}$ \\
\hline Poner en perspectiva & .02 \\
\hline Reinterpretación positiva & $-.27^{* *}$ \\
\hline Focalización en los planes & -.01 \\
\hline Focalización positiva & -.02 \\
\hline
\end{tabular}

Nota: ${ }^{* *} p<.01 ; R^{2}=.46 ; F=21.75$

culturales, sino de naturaleza genética, fisiológica, psicológica y social, que provocan una mayor predisposición en las mujeres a experimentar más ansiedad de diverso tipo, así como sintomatología asociada a esta, es decir, mayor rumiación, mayor preocupación o mayor afectividad negativa. Otros autores (e.g., Blanco et al., 2006) respaldan esta idea de la multifactorialidad en la etiología de la prevalencia de ansiedad entre las mujeres.

Además, se hallaron diferencias en función del género para las estrategias rumiación y culpar a otros, lo cual es similar a lo encontrado por Zlomke y Hahn (2010). Sin embargo, tanto estos autores como Medrano et al. (2013) también reportaron diferencias en la estrategia de poner en perspectiva, lo cual no se replica en nuestros hallazgos. Es probable que esto se deba a que ambos estudios trabajaron con muestras de adultos jóvenes, y no con adolescentes. En este sentido, es dable suponer que la estrategia poner en perspectiva, que supone restarle importancia al evento negativo comparándolo con otros acontecidos en la vida, sea una estrategia que se acentúa según se alcanza mayor edad. Tampoco se halló un uso diferencial en las estrategias focalización positiva y focalización en los planes, lo cual difiere de lo reportado por Öngen (2010). Por último, se encontró un uso diferencial de la estrategia autoculparse, siendo esta mayor en mujeres, lo cual no coincide con hallazgos previos. Es posible suponer que existan diferencias culturales en las muestras utilizadas que expliquen estos resultados. En un futuro, investigaciones transculturales podrían ahondar respecto a esto y dar luz sobre las inconsistencias halladas. Sería interesante analizar si existe una relación entre ciertos patrones de crianza y características socioculturales y el uso de estrategias de regulación emocional en distintas regiones o países.

En relación con los efectos del uso de estrategias cognitivas de regulación emocional sobre los niveles de ansiedad, los resultados sobre el aporte explicativo de las estrategias autoculparse, catastrofización, reinterpretación positiva y rumiación coinciden con lo expuesto por Yousefi (2007) 
y Legerstee et al. (2011), aunque este último estudio también reporta que la estrategia culpar a otros resultó predictoras de los niveles de ansiedad. Si bien se halló una asociación entre dicha estrategia y la ansiedad, la correlación resultó muy baja y fue eliminada en el modelo de regresión. Nuestros resultados también coinciden parcialmente con los de Garnefski et al. (2002), quienes hallaron que rumiación y autoculparse explicaban los niveles de ansiedad. A diferencia de estos autores, aquí también autoculparse y reinterpretación positiva resultaron predictoras en el modelo de regresión. También coincidimos parcialmente con el estudio transcultural de Potthoff et al. (2016) y, desde un enfoque más global, coincidimos con lo expuesto en la literatura en general (Aldao et al., 2010; Cisler et al., 2010; Nolen-Hoeksema, 2012) respecto al aporte del uso de estrategias de regulación emocional sobre los niveles de ansiedad.

No obstante, cabe aclarar que parecería que la ansiedad se relaciona en mayor medida con la frecuencia de uso de estrategias desadaptativas que con el uso poco frecuente de estrategias adaptativas. Si bien la reinterpretación positiva ha resultado predictora en este estudio y en otros similares (e.g., Legerstee et al., 2011), el resto de las estrategias consideradas adaptativas según el modelo de Garnefski et al. (2001) no suelen evidenciar efectos directos sobre los niveles de ansiedad salvo en algunos pocos estudios (e.g., Potthoff et al., 2016). En este sentido, resultan más frecuentes los hallazgos que apuntan al rol perjudicial del uso de estrategias desadaptativas en el incremento de los síntomas y desórdenes de ansiedad, que los que reportan una relación entre la disminución de la ansiedad y el uso de estrategias adaptativas.

Los datos aquí reportados suponen un aporte tanto en términos del interés científico por las relaciones entre las emociones y los trastornos psicopatológicos, como del diseño de intervenciones psicoterapéuticas orientadas a la reducción, disminución y tratamiento de los distintos desórdenes de ansiedad. Además, para la franja etaria analizada, es decir, adolescentes de entre 15 y 18 años, hallazgos como este y otros presentes en la literatura resultan de interés en la conducción de los proyectos pedagógicos y de apoyo al estudiante, con el objeto de reducir los síntomas y trastornos psicopatológicos comunes en dichas edades (Orgilés et al., 2012). Se espera que los aportes colaboren a que orientadores educativos, en particular, y demás actores institucionales, en general, pueden incrementar el bienestar de los estudiantes y conducir mejoras en la práctica educativa.

Finalmente, existen ciertas limitaciones para tener en cuenta. En primer lugar, debe señalarse que trabajar con técnicas de autoinforme siempre supone una restricción en la interpretación de los datos. Aunque las técnicas con las que se ha trabajado tienen una amplia difusión en la literatura y gran cantidad de evidencias sobre su validez y confiabilidad, el uso de medidas directas como herramientas adicionales en la toma de datos hubiera supuesto un respaldo significativo. En segundo lugar, como ya fue mencionado, no se cuenta con datos precisos sobre el funcionamiento de la escala CERQ en adolescentes. Si bien existen antecedentes de que se han utilizado otras versiones de esta en dicha población (e.g. Duarte et al., 2015; Garnefski et al., 2005), es necesario aún conducir estudios sobre las propiedades psicométricas de esta y otras versiones del CERQ con edades comprendidas entre los 12 y los 18 años. En este estudio, el análisis de confiabilidad de la escala reveló un coeficiente por debajo del esperado para la dimensión aceptación. Se observó también que, la eliminación del ítem 25 mejoraba significativamente la confiabilidad de la subescala. Estudios previos han reportado ya un comportamiento precario de dicho ítem en la subescala (e.g., Medrano et al., 2013), o la posibilidad de que la dimensión en sí misma refleje más desesperanza que aceptación del evento negativo (Martin \& Dahlen, 2005). Se sugiere, por lo tanto, que futuras investigaciones sobre la estructura factorial del instrumento y su validez realicen revisiones de dicho ítem y de la dimensión aceptación.

En tercer lugar, dado el tamaño de la muestra, no fue posible evaluar la invarianza factorial del instrumento entre los géneros, lo cual se supone solo tácitamente al analizar las diferencias entre participantes de género femenino y masculino. Un estudio al respecto supondría un gran aporte en la evaluación de la frecuencia de uso de estrategias cognitivas de regulación emocional. Finalmente, dado que los participantes pertenecían íntegramente a escuelas concertadas, la generalización de los resultados se encuentra limitada dadas las características socioeconómicas de estas instituciones educativas. Estudios ulteriores deberían considerar la inclusión de participantes provenientes de distintos niveles socioeconómicos, e incluso evaluar si este último no tiene una incidencia sobre las variables bajo análisis o la relación entre estas.

\section{Referencias}

Aldao, A., Nolen-Hoeksema, S., \& Schweizer, S. (2010). Emotion-regulation strategies across psychopathology: A meta-analytic review. Clinical Psychology Review, 30(2), 217-237. http://dx. doi.org/10.1016/j.cpr.2009.11.004

Andrés, M. L., Castañeiras, C. E., \& Richaud de Minzi, M. C. R. (2015). Contribución de las estrategias cognitivas de regulación emocional en la ansiedad, depresión y bienestar en niños de 10 años de edad. Resultados preliminares. PSIENCIA. Revista Latinoamericana de Ciencia Psicológica, 6(2), 81-89. http://dx. doi.org/10.5872/psiencia/6.2.24

Bender, P. K., Reinholdt-Dunne, M. L., Esbjørn, B. H., \& Pons, F. (2012). Emotion dysregulation and anxiety in children and adolescents: Gender differences. Personality and Individual Differences, 53(3), 284-288. http://dx.doi.org/10.1016/j.paid.2012.03.027

Blanco, C., Hasin, D. S., Petry, N., Stinson, F. S., \& Grant, B. F. (2006). Sex differences in subclinical and DSM-IV pathological gambling: results from the National Epidemiologic Survey on Alcohol and Related Conditions. Psychological Medicine, 36(7), 943-953. http://dx.doi.org/10.1017/S0033291706007410

Caballo, V. E., Salazar, I. C., Arias, B., Calderero, M., Irurtia, M. J., Ollendick, T., \& CISO-A Research Team. (2016). The Social Anxiety Questionnaire for Children: Cross-cultural assessment with a new self-report measure. Journal of Psychopathology and Behavioral Assessment, 38(4), 695-709. http://dx.doi.org/10.1007/ s10862-016-9562-x

Caballo, V. E., Salazar, I. C., Irurtia, M. J., Arias, B., Hofmann, S. G., \& CISO-A Research Team. (2014). Differences in social anxiety between men and women across 18 countries. Personality and Individual Differences, 64, 35-40. http://dx.doi.org/10.1016/j. paid.2014.02.013

Cano-Vindel, A. (2011). Los desórdenes emocionales en atención primaria. Ansiedad y Estrés, 17(1), 75-97.

Cisler, J. M., Olatunji, B. O., Feldner, M. T., \& Forsyth, J. P. (2010). Emotion regulation and the anxiety disorders: An integrative review. Journal of Psychopathology and Behavioral Assessment, 32(1), 68-82. http://dx.doi.org/10.1007/s10862-009-9161-1 
Copeland, W. E., Angold, A., Shanahan, L., \& Costello, E. J. (2014). Longitudinal patterns of anxiety from childhood to adulthood: The Great Smoky Mountains Study. Journal of the American Academy of Child \& Adolescent Psychiatry, 53(1), 21-33. http:// dx.doi.org/10.1016/j.jaac.2013.09.017

Díaz Santos, C., \& Santos Vallín, L. (2018). La ansiedad en la adolescencia. Enfermería Comunitaria, 6, 7-10.

Domínguez-Lara, S. A. (2017). Influencia de las estrategias cognitivas de regulación emocional sobre la ansiedad y depresión en universitarios: análisis preliminar. Salud Uninorte, 33(3), 315-321.

Domínguez-Lara, S. A., \& Medrano, L. (2016). Propiedades psicométricas del cuestionario de regulación cognitiva de la emociones (CERQ) en estudiantes universitarios de Lima. Psychologia, 10(1), 53-67. http://dx.doi.org/10.21500/19002386.2466

Domínguez-Lara, S. A., Alarcón-Parco, D., \& Navarro-Loli, J. S. (2017). Relación entre ansiedad ante exámenes y rendimiento en exámenes en universitarios: análisis preliminar de la diferencia según asignatura. Revista Digital de Investigación en Docencia Universitaria, 11(1), 166-176. http://dx.doi.org/10.19083/ridu.11.492

Domínguez-Sánchez, F. J., Lasa-Aristu, A., Amor, P. J., \& Holgado-Tello, F. P. (2013). Psychometric properties of the Spanish version of the Cognitive Emotion Regulation Questionnaire. Assessment, 20(2), 253-261. http://dx.doi.org/10.1177/1073191110397274

Duarte, A. C., Matos, A. P., \& Marqués, C. (2015). Cognitive emotion regulation strategies and depressive symptoms: Gender's moderating effect. Procedia-Social and Behavioral Sciences, 165, 275-283. http://dx.doi.org/10.1016/j.sbspro.2014.12.632

Eftekhari, A., Zoellner, L. A., \& Vigil, S. A. (2009). Patterns of emotion regulation and psychopathology. Anxiety, Stress, \& Coping, 22(5), 571-586. http://dx.doi.org/10.1080/10615800802179860

Fonseca-Pedrero, E., Paino, M., Sierra-Baigrie, S., Lemos-Giráldez, S., \& Muñiz, J. (2012). Propiedades psicométricas del Cuestionario de ansiedad estado-rasgo (STAI) en universitarios. Psicología Conductual, 20(3), 547-561.

Garnefski, N., \& Kraaij, V. (2007). The Cognitive Emotion Regulation Questionnaire psychometric features and prospective relationships with depression and anxiety in adults. Cognitive Emotion Regulation European Journal of Psychological Assessment, 23(3), 141-149. http://dx.doi.org/10.1027/1015-5759.23.3.141

Garnefski, N., Kraaij, V., \& Spinhoven, P. (2001). Negative life events, cognitive emotion regulation and emotional problems. Personality and Individual Differences, 30(8), 1311-1327. http:// dx.doi.org/10.1016/S0191-8869(00)00113-6

Garnefski, N., Kraaij, V., \& Van Etten, M. (2005). Specificity of relations between adolescents' cognitive emotion regulation strategies and internalizing and externalizing psychopathology. Journal of Adolescence, 28(5), 619-631. http://dx.doi.org/10.1016/j. adolescence.2004.12.009

Garnefski, N., Legerstee, J., Kraaij, V., Van den Kommer, T., \& Teerds, J. A. N. (2002). Cognitive coping strategies and symptoms of depression and anxiety: A comparison between adolescents and adults. Journal of Adolescence, 25(6), 603-611. http:// dx.doi.org/10.1006/jado.2002.0507

Garnefski, N., Teerds, J., Kraaij, V., Legerstee, J., \& Van den Kommer, T. (2004). Cognitive emotion regulation strategies and depressive symptoms: Differences between males and females. Personality and Individual Differences, 36(2), 267-276. http://dx.doi.org/10.1016/S0191-8869(03)00083-7

Gross, J. J. (2014). Emotion regulation: Conceptual and empirical foundations. En J. J. Gross (Ed.), Handbook of emotion regulation (2. ${ }^{a}$ ed., pp. 3-24). Nueva York: Guilford Press.

Guillén-Riquelme, A., \& Buela-Casal, G. (2011). Actualización psicométrica y funcionamiento diferencial del ítem en el State Trait Anxiety Inventory (STAI). Psicothema, 23, 510-515.
Hernández Sampieri, R., Fernández Collado, C., \& Baptista Lucio, P. (2014). Metodología de la Investigación (6. ${ }^{\mathrm{a}}$ ed.). México: McGraw Hill.

Hernández-Pozo, M. del R., Ramírez-Guerrero, N., López-Cárdenas, S. V., \& Macías-Martínez, D. (2015). Relación entre ansiedad, desempeño y riesgo de deserción en aspirantes a bachillerato. Psychologia. Avances de la Disciplina, 9(1), 45-57.

Hervás, G. (2011). Psicopatología de la regulación emocional: el papel de los déficits emocionales en los trastornos clínicos. Psicología Conductual, 19(2), 347-372.

Irurtia, M. J., Caballo, V. E., \& Ovejero, A. (2009). Trastorno de ansiedad provocado por sustancias psicoactivas. Psicología Conductual, 17(1), 155-168.

Jacobs, S. E., \& Gross, J. J. (2014). Emotion regulation in education. Conceptual foundations, current applications, and future directions. En R. Pekrun \& L. Linnenbrink-García (Eds.), International handbook of emotions in educations (pp. 183-201). Nueva York, Londres: Routledge.

Jermann, F., Van der Linden, M., d'Acremont, M., \& Zermatten, A. (2006). Cognitive Emotion Regulation Questionnaire (CERQ). European Journal of Psychological Assessment, 22(2), 126-131. http://dx.doi.org/10.1027/1015-5759.22.2.126

Koole, S. L. (2009). The psychology of emotion regulation: An integrative review. Cognition and Emotion, 23(1), 4-41. http:// dx.doi.org/10.1080/02699930802619031

Leach, L. S., Christensen, H., Mackinnon, A. J., Windsor, T. D., \& Butterworth, P. (2008). Gender differences in depression and anxiety across the adult lifespan: the role of psychosocial mediators. Social Psychiatry and Psychiatric Epidemiology, 43(12), 983-998. http://dx.doi.org/10.1007/s00127-008-0388-z

Ledesma, R., Macbeth, G., \& Cortada de Kohan, N. (2009). Computing effect size measures with ViSta-The visual statistics system. Tutorials in Quantitative Methods for Psychology, 5(1), 25-34. http://dx.doi.org/10.20982/tqmp.05.1.p025

Legerstee, J. S., Garnefski, N., Verhulst, F. C., \& Utens, E. M. (2011). Cognitive coping in anxiety-disordered adolescents. Journal of Adolescence, 34(2), 319-326. http://dx.doi.org/10.1016/j.adolescence.2010.04.008

McLean, C. P., \& Anderson, E. R. (2009). Brave men and timid women? A review of the gender differences in fear and anxiety. Clinical Psychology Review, 29(6), 496-505. http://dx.doi. org/10.1016/j.cpr.2009.05.003

McLean, C. P., Asnaani, A., Litz, B. T., \& Hofmann, S. G. (2011). Gender differences in anxiety disorders: Prevalence, course of illness, comorbidity and burden of illness. Journal of Psychiatric Research, 45(8), 1027-1035. http://dx.doi.org/10.1016/j.jpsychires.2011.03.006

Medrano, L. A., Moretti, L., Ortiz, Á., \& Pereno, G. (2013). Validación del Cuestionario de regulación emocional cognitiva en universitarios de Córdoba, Argentina. PSYKHE, 22(1), 83-96. http:// dx.doi.org/10.7764/psykhe.22.1.473

Mulligan, K., \& Scherer, K. R. (2012). Toward a working definition of emotion. Emotion Review, 4(4), 345-357. http://dx.doi.org/ $10.1177 / 1754073912445818$

Nolen-Hoeksema, S. (2012). Emotion regulation and psychopathology: The role of gender. Annual Review of Clinical Psychology, 8, 161187. http://dx.doi.org/10.1146/annurev-clinpsy-032511-143109

Nolen-Hoeksema, S., Wisco, B. E., \& Lyubomirsky, S. (2008). Rethinking rumination. Perspectives on Psychological Science, 3, 400-424. http://dx.doi.org/10.1111/j.1745-6924.2008.00088.x

Öngen, D. E. (2010). Cognitive emotion regulation in the prediction of depression and submissive behavior: Gender and grade level differences in Turkish adolescents. Procedia-Social and Behavioral Sciences, 9, 1516-1523. 
Orgilés, M., Méndez, X., Espada, J. P., Carballo, J. L., \& Piqueras, J. A. (2012). Síntomas de trastornos de ansiedad en niños y adolescentes: diferencias en función de la edad y el sexo en una muestra comunitaria. Revista de Psiquiatría y Salud Mental, 5(2), 115-120. http://dx.doi.org/10.1016/j.rpsm.2012.01.005

Oros, L. B., Manucci, V., \& Richaud-de Minzi, M. C. (2011). Desarrollo de emociones positivas en la niñez. Lineamientos para la intervención escolar. Educación y Educadores, 14(3), 493-509.

Pekrun, R., \& Linnenbrink-García, L. (2012) Academic emotions and student engagement. En S. L. Chistenson, A. L. Reschly, \& C. Wylie (Eds.), Handbook of research on student engagement (pp. 259-292). Nueva York: Springer.

Potthoff, S., Garnefski, N., Miklósi, M., Ubbiali, A., Domínguez-Sánchez, F. J., Martins, E. C., ... \& Kraaij, V. (2016). Cognitive emotion regulation and psychopathology across cultures: A comparison between six European countries. Personality and Individual Differences, 98, 218-224. http://dx.doi.org/10.1016/j.paid.2016.04.022

Ranta, K., Kaltiala-Heino, R., Koivisto, A. M., Tuomisto, M. T., Pelkonen, M., \& Marttunen, M. (2007). Age and gender differences in social anxiety symptoms during adolescence: The Social Phobia Inventory (SPIN) as a measure. Psychiatry Research, 153(3), 261270. http://dx.doi.org/10.1016/j.psychres.2006.12.006

Sheppes, G., Suri, G., \& Gross, J. J. (2015). Emotion regulation and psychopathology. Annual Review of Clinical Psychology, 11(1), 379405. http://dx.doi.org/10.1146/annurev-clinpsy-032814-112739

Spielberger, C. D., Gorsuch, R. L., \& Lushene, R. E. (1970). Manual for the state-trait anxiety inventory. California: Consulting Psychologists Press.
Spielberger, C. D., Gorsuch, R. L., Lushene, R. E., \& Cubero, N. S. (1999). STAl: Cuestionario de ansiedad estado-rasgo: manual. Madrid: TEA.

Xu, Y., Schneier, F., Heimberg, R. G., Princisvalle, K., Liebowitz, M. R., Wang, S., \& Blanco, C. (2012). Gender differences in social anxiety disorder: Results from the national epidemiologic sample on alcohol and related conditions. Journal of Anxiety Disorders, 26(1), 12-19. http://dx.doi.org/10.1016/j.janxdis.2011.08.006

Yousefi, F. (2007). The relationship of cognitive emotion regulation strategies with depression and anxiety in students of special middle schools for talented students in Shiraz. Journal of Exceptional Children (Research on Exceptional Children), 4(22), 871-892.

Zelazo, P. D., \& Cunningham, W. A. (2007). Executive function: Mechanisms underlying emotion regulation. En J. J. Gross (Ed.), Handbook of emotion regulation (pp. 135-158). Nueva York: Guilford Press.

Zhu, X., Auerbach, R. P., Yao, S., Abela, J. R., Xiao, J., \& Tong, X. (2008). Psychometric properties of the cognitive emotion regulation questionnaire: Chinese version. Cognition \& Emotion, 22(2), 288-307. http://dx.doi.org/10.1080/02699930701369035

Zlomke, K. R., \& Hahn, K. S. (2010). Cognitive emotion regulation strategies: Gender differences and associations to worry. Personality and Individual Differences, 48(4), 408-413. http:// dx.doi.org/10.1016/j.paid.2009.11.007 